\title{
ESTIGMA O REALIDAD: ¿EFICIENCIA DE LAS EMPRESAS PÚBLICAS? ANÁLISIS DE CASOS PERUANOS Y MEXICANOS
}

\author{
STIGMA OR REALITY: EFFICIENCY OF PUBLIC COMPANIES? \\ ANALYSIS OF PERUVIAN AND MEXICAN CASES
}

Luz María Farías Ruelas Universidad de Guadalajara Jalisco, México

Correo electrónico: luzmariafarias@gmail.com

Cleofé Maritza Verástegui Corrales Universidad Nacional Mayor de San Marcos Lima, Perú ORCID: http://orcid.org/0000-0002-2560-3831 Correo electrónico: cverasteguic@unmsm.edu.pe

Emilio Gustavo Bobbio Rosas Universidad Nacional Mayor de San Marcos Lima, Perú

ORCID: http://orcid.org/0000-0002-1568-7357

Correo electrónico: ebobbior@unmsm.edu.pe

[Recibido: 01/07/2019 Aceptado: 02/08/2019 Publicado: 28/08/2019]

\section{RESUMEN}

En las últimas dos décadas, se ha implementado en América Latina la gestión basada en resultados para los entes públicos, buscando incrementar eficiencia. La investigación tiene como propósito identificar para Perú y México, los resultados del impacto directo en la eficiencia de la aplicación del recurso público, visto desde un incremento directo a los bienes y servicios que se ofrecen a los ciudadanos. Servicios como trámites directos en las dependencias gubernamentales, entre otros; bienes, derechos o subsidios, otorgados directamente a los ciudadanos: alumbrado, programas de apoyo a grupos con extrema pobreza, vialidades, entre otros. El estudio se va enfocar en mitos y realidades, intereses políticos, y la presentación de una serie de medidas que conducirían a la optimización de las empresas públicas; toda vez que, en otros continentes, sí existe un beneficio directo a la sociedad por contar con empresas públicas eficientes. Incluso en América Latina, han existido y existen empresas que desmienten el dicho popular por su rentabilidad, entendida como un beneficio directo a la sociedad; ya sea a través de ingresos públicos que son destinados a bienes y servicios públicos directos en las ciudades, o por su calidad y eficiencia en la atención y seguimiento a los trámites ingresados por los ciudadanos. Quizás la ineficiencia de los entes públicos no constituya una realidad, sino una falacia construida por entes interesados en que se vuelva una verdad incontrastable.

\begin{abstract}
In the last two decades, has been implemented in Latin America results based management for public entities, searching to increase efficiency. The research has as purpose to identify for Peru and Mexico, the results of the direct impact in the efficiency of the application of public resources, seen from a direct increase in the goods and services offered to the citizens. Services as a direct procedures in government agencies, among others; goods, rights or subsidies, granted directly to the citizens: lighting, support programs for groups with extreme poverty, roads, among others. It will focus on myths and realities, political interests, and the presentation of a series of measures that could lead to the optimization of public companies; every time that, in other continents, it exist a direct benefit to the society by had efficient public companies. Even in Latin America, have been exist and exist companies that deny the popular saying for its profitability, understood as a direct benefit to the society; either through public revenues that are destinated to direct public goods and services in the cities, or by their quality and efficiency in the atention and tracing of the procedures entered by citizens. Perhaps the inefficiency of public entities does not establish a reality, but a fallacy built by entities interested in turnnig an incontrastable truth.
\end{abstract}

Keywords: Management; public resource; efficiency; stigma; company.
Palabras clave: Gestión; recurso público; eficiencia; estigma; empresa.

( - Los autores. Este artículo es publicado por la Revista Quipukamayoc, Universidad Nacional Mayor de San Marcos. Este es un artículo de acceso abierto, distribuido bajo los términos de la Licencia Creative Commons Atribución-NoComercial-Compartirlgual 4.0 Internacional.(http://creativecommons.org/licenses/by-nc-sa/4.0/), que permite el uso no comercial, distribución y reproducción en cualquier medio, siempre que la obra original sea debidamente citadas. 


\section{INTRODUCCIÓN}

Según el Ministerio de Economía y Finanzas (MEF) del Perú, al 2017 existían 119 empresas públicas operativas, 20 no operativas y 21 en proceso de liquidación; su valor del capital bordea los 18520 millones de soles. De este universo, un grupo se encuentra regulado por el Fondo Nacional de Financiamiento de la Actividad Empresarial del Estado (FONAFE) y otro grupo está regulado por la Dirección General de Presupuesto Público (DGPP) del Ministerio de Economía y Finanzas (MEF). Estas entidades reguladoras, en el marco de su competencia, dirigen y regulan el proceso presupuestario de las empresas del Estado. En el Perú, el MEF promueve y lidera la mejora continua de la Gestión de las Finanzas Públicas (GFP) en todos los niveles de gobierno, actualmente, este trabajo lo viene realizando con el apoyo de diversos donantes, entre ellos la Cooperación Suiza-SECO desde octubre del 2015, fecha en la cual la Cooperación Suiza-SECO firmó 11 acuerdos con distintas entidades dentro del Programa de Fortalecimiento de la Gestión de las Finanzas Públicas a Nivel Subnacional para el Desarrollo de una Gestión Descentralizada (2015-2019).

Sin embargo, hasta la fecha, a la luz de lo aparecido en la prensa, debidamente sustentado y documentado, los resultados han sido muy pobres en lo que se refiere a una gestión correcta de las finanzas públicas desde un punto de vista ético y moral; según el Índice de Percepción de la Corrupción (IPC) elaborado por Transparencia Internacional (TI), desde el 2012 el Perú no había estado en un puesto tan bajo, el 2015 estuvimos en el puesto 88 de 180 naciones; el 2016, en el puesto101; el 2017, en el puesto 96 y el 2018, en el puesto 105.
El Programa de Mejora Continua de la Gestión de las Finanzas Públicas (PMC) fue implementado por el Basel Institute on Governance (BIG) y brinda asistencia técnica en los gobiernos regionales y a las municipalidades provinciales inmersas en el programa. Dentro del marco del programa se ha financiado la elaboración de evaluaciones de la GFP, siguiendo la metodología internacionalmente aceptada sobre Public Expenditure and Financial Accountability (PEFA), que sirve como referencia para la identificación de fortalezas y debilidades en GFP, y mejorar así el punto focal y prioridades de la asistencia técnica en beneficio de los gobiernos participantes.

En el mismo orden de ideas, el Instituto Mexicano para la Competitividad (IMCO) genera el índice de Riesgo de Corrupción dentro del sistema mexicano de contrataciones públicas, la información respecto a las Unidades Compradoras (UC), entendiendo estas como empresas públicas, respecto al resultado publicado en la Ciudad de México el pasado 6 de marzo 2018, muestra de un rango de 500 UC las que inciden con un porcentaje elevado de corrupción (ver tabla 1).

Se puede observar que el índice de riesgo de corrupción es elevado, no debería existir en una empresa pública la sensación de corrupción, por parte de la sociedad. Peor aún, si éstas brindan servicios básicos como lo son la salud (ISSTE, IMSS), comunicación (SCT, Aeropuertos y SA) o incluso aquellas dedicadas a entregar el producto de programas sociales como lo es Prospera.

Por otro lado, la Comisión Económica para América Latina y el Caribe (CEPAL), donde CEPAL (2007) realiza un análisis de países con mayor incidencia de corrupción. Dentro de los países que fluctúan entre el 16 y $40 \%$ en el

Tabla 1

Dependencias o entidades más riesgosas.

\begin{tabular}{llccc}
\hline $\mathrm{N}^{\circ}$ & $\begin{array}{c}\text { Las 10 dependencias o entidades } \\
\text { con más UC en riesgo }\end{array}$ & $\begin{array}{c}\text { Número de UC en } \\
\text { riesgo }\end{array}$ & $\begin{array}{c}\text { UC en riesgo (\% del total en la } \\
\text { dependencia o entidad) }\end{array}$ & $\begin{array}{c}\text { Monto de la dependencia o entidad } \\
\text { (pesos mexicanos) 2012-2017 }\end{array}$ \\
\hline 1 & CFE & 91 & $25 \%$ & 424466885322 \\
2 & ISSSTE & 46 & $53 \%$ & 147627542114 \\
3 & IMSS & 42 & $38 \%$ & 401055667795 \\
4 & Aeropuertos y SA & 28 & $47 \%$ & 6319534861 \\
5 & SCT & 18 & $27 \%$ & 327324355171 \\
6 & Prospera & 17 & $52 \%$ & 4539204482 \\
7 & Liconsa & 14 & $52 \%$ & 19477741036 \\
8 & CNDPI & 11 & $35 \%$ & 6223063582 \\
9 & SAGARPA & 10 & $30 \%$ & 4556218295 \\
10 & SEMARNAT & 9 & $53 \%$ & 2692089607 \\
\hline
\end{tabular}

Fuente: Instituto Mexicano para la Competitividad (IMCO), mayo del 2019. 
que se paga soborno para cualquier tipo de trámite, se encuentran México y Perú.

Si los servicios que brindan las empresas públicas a la sociedad se consideran gratuitos o con un costo solo de recuperación, la eficiencia se estaría midiendo en el grado de confianza de la sociedad por el trámite realizado, de forma expedita y sin requisitos engorrosos. Desde ahí se observa la poca eficiencia de las empresas públicas en México y Perú.

Considerando que el enfoque de la investigación es descriptivo, se ha utilizado la información financiera del Fondo Nacional de Financiamiento de la Actividad Empresarial del Estado (FONAFE) que publica el Ministerio de Economía y Finanzas del Perú, además de los estados financieros del Gobierno Federal del Estado Mexicano.

\section{Empresas del Estado Peruano}

Abusada, Cusato y Pastor (2008), realizaron un resumen sobre la actividad empresarial del Estado Peruano señalando que:

Se inició hace cuatro décadas en el Perú y llegó a su estado más crítico a fines de la década de los ochenta. En ese momento, las empresas públicas constituían una de las principales fuentes del desorden fiscal existente y la ineficiencia de los mercados (controles de precios y otras distorsiones), que llevó al país a una hiperinflación sin precedentes. El valor actual de las pérdidas acumuladas de las empresas públicas a 1990 ascendía a US\$17 738 millones (67\% del PBI de dicho año), un valor muy similar al de la deuda pública externa a la fecha (US\$18 934 millones). Las dictaduras militares de la década de los setenta (1968-1980) y luego los dos gobiernos de la siguiente década (1980-1990) originaron un fuerte aumento en el número de empresas públicas, que pasaron de 40 en 1968 a 186 en el año 1990, de las cuales el 73\% aproximadamente, se dedicaba a actividades no financieras. En 1990, el Estado participaba en el $100 \%$ de la generación de electricidad, agua potable y comunicaciones, así como la producción de petróleo y gas (Alvarez,1991). La situación cambió en la década de los noventa, con el inicio de las privatizaciones de empresas y concesiones, así como con el reordenamiento y liquidación de la mayoría de las empresas existentes (p.1).

Como vemos, el país cuenta con un adecuado marco legal, con los sistemas y demás herramientas informáticas que facilitan la Gestión de las Finanzas Públicas, además de un sistema educativo que presenta universidades de gran calidad, que ofrecen cursos de pregrado, maestrías y doctorados y el resultado de estos estudios es demos- trado por la cantidad de peruanos que ocupan puestos de responsabilidad en instituciones financieras de nivel y significación mundial.

Aparecen entonces las siguientes preguntas:

- ¿Hay necesidad de empresas estatales?

- ¿Por qué en el Perú las empresas privadas triunfan donde las estatales fracasan?

La respuesta a la primera pregunta ha sido contestada en múltiples ocasiones, pero un análisis completo lo encontramos en el artículo ¿Necesitamos empresas estatales?, se resume en que la empresa privada busca la máxima ganancia y no el bienestar del pueblo, por lo que se requieren en algunas áreas estratégicas y que, si se privatizan las empresas sin un adecuado control, puede llegar a crearse un problema económico para la población, en vez de una solución. Compartimos esa opinión, más aún en un sistema tan sucio que todos los expresidentes afrontan serias, documentadas y evidentes acusaciones por actos de corrupción, originadas por su ambición y, claro, la tentación generada por la empresa privada.

La segunda pregunta que en el fondo es el meollo de nuestra investigación tiene una primera respuesta simple; la corrupción, ya que en las empresas privadas esta disminuye porque los mismos dueños no van a robar su empresa; $y$, por otra parte, las empresas tienen controles más estrictos, en tanto que muchos peruanos no sienten el Estado o la empresa que están dirigiendo como suya.

Pero esta segunda parte de la respuesta nos conduciría a una nueva pregunta ¿no hay gente capaz y comprometida que pueda asumir la dirección de las empresas estatales? Y esta pregunta requiere de una respuesta compleja:

- Si los presidentes (que son los que nombran directa o interpósita persona a esos gerentes) han demostrado todos ser corruptos, ¿a qué clase de personas necesitan en esos puestos?

- ¿Quién garantiza la permanencia en el puesto a una persona que deja una carrera brillante para asumir una función de servicio al país?, la respuesta es nadie y eso motiva una gran rotación de directivos que es otra causa del desgobierno de esas empresas.

A fin de determinar la salud de la empresa de FONAFE motivo de la investigación, revisamos el Estado de Resultados Integrales del año 2017 versus el año 2016 y observamos que los ingresos de las ventas de bienes y prestación de servicios se ven disminuidos por los gastos de administración que se incrementaron en S/.386 047,3 con relación al año anterior, seguido de Otros Gastos Operativos (ver tabla 2). 


\section{La Gestión de las Finanzas Públicas en México}

México inicia la migración de una transparencia y fiscalización en la aplicación de los recursos públicos en el ejercicio 2000, por ser una facultad plasmada en los artículos 25 y 26 de la Constitución Política de los Estados Unidos Mexicanos (CEPEUM) para la intervención en la economía. Artículos que establecen la rectoría del desarrollo nacional y la obligación de planificarla, adicional a ello, con los tratados internacionales que ha firmado México, es obligatorio generar un Plan Nacional de Desarrollo que incluya políticas públicas para garantizar un crecimiento económico, inclusión de grupos vulnerables y principalmente una aplicación y resultado de los recursos públicos, que permitan identificar el buen desempeño de las empresas públicas y las diversas secretarías, dando muestra clara de que son utilizados de forma eficiente.

El Plan Nacional de Desarrollo (PND) en su versión 2019-2024, bajo el liderazgo del Presidente de la República Mexicana, señala que la CPEUM establece, como parte de sus directrices, la planeación democrática del desarrollo nacional como el instrumento que articula las políticas públicas para la Gobernanza Nacional, pero también como la fuente directa de la democracia participativa a través de mecanismos de consulta con la sociedad. Dando como resultado que el desarrollo nacional es tarea de todos.

Dentro del mismo documento, se hace referencia a la observancia y el cumplimiento del marco legal, es decir, el cumplimiento de la CPEUM y la Ley de Planeación, garantizando la integridad y sustentabilidad a través de principios rectores como son la honradez y honestidad, no al gobierno rico con pueblo pobre, al margen de la ley nada, por encima de la ley nadie, economía para el bienestar, entre otros que contribuyan a mejorar la equidad social y el bienestar de las familias mexicanas.

Cada sexenio presidencial, emite su estrategia de trabajo que deberá estar alineada a los objetivos internacionales de desarrollo, así como avanzar en materia de combate a la corrupción y transparencia en el uso y destino de los recursos públicos. Por ello, y a manera de cronograma, en la figura 1 se muestra la estructura jurídica mexicana para la transparencia del uso y destino de los fondos públicos, a partir del año 2000 hasta el ejercicio 2018, donde se puede observar que las diversas leyes aprobadas tienen una tendencia marcada, para generar una eficiencia en los recursos públicos.

La realidad vivida en México en los últimos 20 años se presenta con las empresas públicas administradas de forma ineficiente y, como el caso de Petróleos Mexicanos (PEMEX), los recursos obtenidos de forma adicional son utilizados en la economía nacional para subsanar el déficit de la recaudación fiscal. Es decir, se retira el recurso de la empresa para trasladarlo a la Secretaría de Hacienda y Crédito Público (SHCP) y sea distribuido en las partidas presupuestales previamente autorizadas por el Congreso de la Unión Mexicana.

El retirar el recurso de las empresas paraestatales, paramunicipales, organismos públicos descentralizados, entre otros, genera un desequilibrio en las finanzas de éstos, motivo por el cual se hizo necesario reformar leyes e incluso emitir nuevas leyes, con el fin de transparentar el uso del dinero público, generar una aplicación eficiente y

Tabla 2

Estado de Resultados Integrales (miles de soles).

\begin{tabular}{lrr}
\hline CONCEPTO & 2017 & 2016 \\
\hline Ventas Netas de Bienes & 5542329,8 & 4635217,9 \\
Prestación de Servicios & 6837167,1 & 6554396,2 \\
Ingresos por intereses & 2647738,2 & 2744124,1 \\
TOTAL DE INGRESOS DE ACTIVIDADES ORDINARIAS & 15027235,1 & 13933738,2 \\
Costo de Ventas & 8556523,4 & 7456061,8 \\
Gastos por intereses & 860189,9 & 812455,9 \\
GANANCIA O PÉRDIDA BRUTA & 5610521,8 & 5665220,5 \\
Gastos de ventas y distribución & 587374,5 & 563786,7 \\
Gastos de administración & 2570655,9 & 2184608,6 \\
Otros Ingresos Operativos & 513691,8 & 505165,6 \\
Otros Gastos Operativos & 1188642,9 & 1049149,0 \\
GANANCIA O PÉRDIDA OPERATIVA & 1777540,3 & 2372841,8 \\
\hline
\end{tabular}

Fuente: Elaboración propia con información del Ministerio de Economía y Finanzas (MEF). 


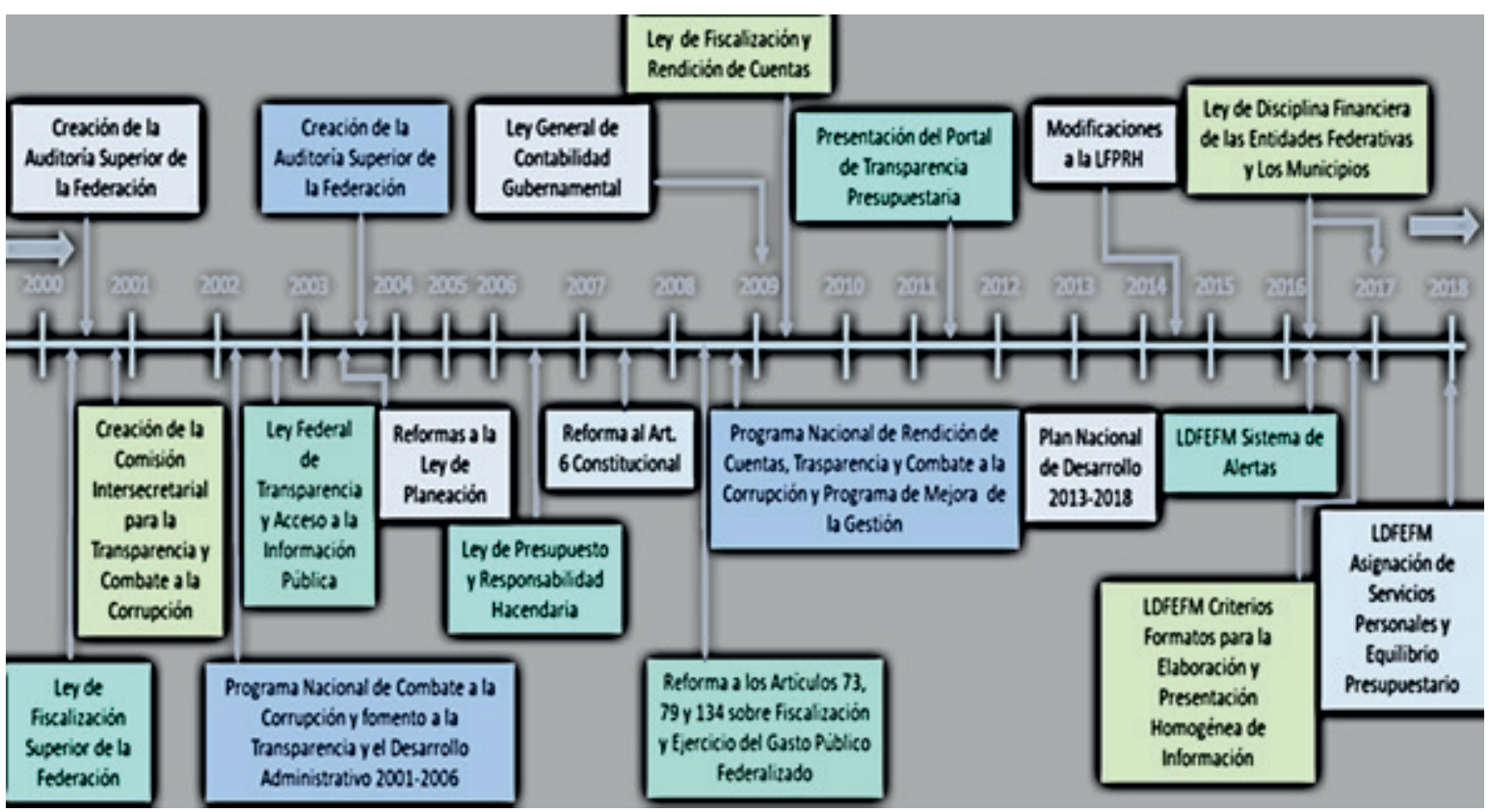

Figura 1. Marco Legal Mexicano.

Fuente: Secretaría de la Hacienda Pública. (2019). Matriz de indicadores (Material del curso). Secretaría de Medio Ambiente y Desarrollo Territorial, Estado de Jalisco, México

establecer reglas de comprobación para cada supuesto de aplicación de recursos.

Leyes y organismos públicos, como la Auditoría Superior de la Federación, la Comisión Intersecretaría para la Transparencia y Combate a la corrupción, Ley de Presupuesto y Responsabilidad Hacendaria, Ley de Disciplina Financiera, hacen pensar que las empresas públicas deben ser eficientes en su administración; sin embargo, año con año los resultados de la administración de las empresas públicas muestran que no existe una administración eficiente.

Dentro del listado de empresas públicas mexicanas o de participación de recurso público en el ámbito federal, que suman un total de 200 de conformidad con el dato publicado el 15 de agosto de 2018 en el Diario Oficial de la Federación, podemos encontrar las siguientes 19 empresas, que se puede decir son las más importantes: 1. Petróleos mexicanos, 2. Comisión Federal de Electricidad, 3. Instituto del Fondo Nacional de la Vivienda para los Trabajadores, 4. Aeropuertos y Servicios Auxiliares, 5. Caminos y Puentes Federales de Ingresos y Servicios Conexos, 6. Nacional Financiera, 7. Fondo de Vivienda del Instituto de Seguridad y Servicios de los Trabajadores del Estado, 8. Banco Nacional de Comercio Exterior, 9. Pronósticos para la Asistencia Pública, 10. Leche Industrializada Conasupo. S.A. de C.V., 11. Lotería Nacional para la Asistencia Pública, 12. Sistema de Transporte Colectivo, 13. Banco Nacional del Ejército,
Fuerza Aérea y Armada, 14. Telecomm-Telégrafos, 15. Correos de México, 16. Financiera Rural, 17. Banco de Desarrollo Rural, S.A., 18. AGROASEMEX, 19. Instituto Mexicano del Seguro Social. Es de analizar que la mayoría de estas empresas públicas, año con año han reflejado ingresos insuficientes para su operación y que sus estados financieros, no se presentan de forma clara.

En México la Ley Federal de Presupuesto y Responsabilidad Hacendaria (LFPRH) establece que todo aumento del déficit debe ser justificado y que en un lapso de 3 años deberá recuperarse el equilibrio fiscal.

A manera de ejemplo, de conformidad con el informe financiero del cuarto trimestre del 2018, Petróleos Mexicanos (2019) identifica la siguiente información:

Utilidad integral: Se registró una utilidad integral de MXN 114,1 mil millones, principalmente como resultado de un incremento de MXN 220,7 mil millones en las ganancias actuariales por beneficios a empleados, dada la actualización de la tasa de descuento que pasó de 7,89\% en 2017 a 9,29\% en 2018. Evolución del rendimiento (pérdida) integral (MXN millones) $(299,759) \quad(125,544) \quad 172,826 \quad 11,973$ $(4,686)$ 77,417 $221(83,095) 4 T 17$ Rendimiento de operación, intereses netos pagados, costo por derivados financieros, pérdida en cambios, efecto de asociadas, impuestos $y$ derechos 4 T18 $(278,847)$ 
$114,112174,215218,2884 T 17$, rendimiento neto, otros resultados integrales 4 T18 (p.8)

Sin embargo, aún y con la utilidad integral reportada, se incrementa la deuda; según datos emitidos por la propia empresa pública: la deuda financiera total incrementó $2,2 \%$ comparada con el cierre del año pasado, debido al efecto del tipo de cambio en el periodo (Petróleos Mexicanos, 2019).

Si bien es cierto, las empresas de capital privado realizan apalancamiento con recursos externos para hacer eficiente el recurso obtenido; también lo es dentro del ámbito privado, ya que se obtienen resultados que se ven reflejados en un incremento de capacidad productiva, lo que no se ha visto en el caso de Petróleos Mexicanos, o Comisión Federal de Electricidad o empresas consideradas públicas. Tal es el caso del primer trimestre del ejercicio 2019, que en abasto de combustible, México sufrió una demora considerable provocando un caos en los diversos sectores productivos del país.

Desde la presentación de los informes financieros de las empresas públicas para la consolidación de la información del Gobierno Federal, no es claro si se obtiene un superávit o déficit de la administración de las empresas públicas, solo se presenta en comparación con un ejercicio, que es el inmediato anterior.

Se revisará el resultado de cada una de las 200 empresas públicas, para determinar cuál de ellas obtiene un superávit o déficit, adicional a ello identificar la eficiencia en la aplicación del recurso público, trasladada a servicios directos a la sociedad. En el mejor de los casos, la información está desactualizada, no muestra la información del ejercicio 2019, ni la del ejercicio 2018. Es frecuente que no se encuentre la información financiera publicada, cual obligación jurídica se tiene.

A continuación, se muestra el informe de estados financieros del Gobierno Federal, específicamente, en su apartado de Patrimonio de organismos descentralizados de control presupuestal indirecto por el ejercicio 2011, expresado en millones de pesos. Se puede identificar que sólo muestra los movimientos realizados entre el ejercicio 2010 y 2011 (ver tabla 3 ).

\section{CONCLUSIONES}

Se puede observar que, en materia jurídica, se ha trabajado en la transparencia del uso y destino del dinero público, que tengan una justificación y con ello hacer que las Empresas Públicas sean eficientes.

Tabla 3

Estados Financieros del Gobierno Federal (millones de pesos mexicanos).

\begin{tabular}{|c|c|c|c|c|}
\hline DEPENDENCIAS Y ORGANISMOS & $\begin{array}{c}\text { SALDO } \\
2010 \\
\end{array}$ & $\begin{array}{c}\text { MOV. } \\
\text { PRESUPUESTARIO }\end{array}$ & $\begin{array}{c}\text { MOV. } \\
\text { POR AJUSTE }\end{array}$ & $\begin{array}{l}\text { SALDO } \\
2011 \\
\end{array}$ \\
\hline TOTAL & 133850,9 & 9204,4 & $(183,0)$ & 142872,3 \\
\hline HACIENDA Y CRÉDITO PÚBLICO & 10865,7 & 1490,1 & 0,0 & 12355,8 \\
\hline Servicio de Administración y Enajenación de Bienes & 4718,9 & & 0,0 & 4718,9 \\
\hline Financiera Rural & 3819,5 & 1384,3 & 0,0 & 5203,8 \\
\hline Comisión Nacional para el Desarrollo de los Pueblos Indígenas & 1381,7 & 80,3 & 0,0 & 1462,0 \\
\hline Otros & 945,6 & 25,5 & 0,0 & 971,1 \\
\hline COMUNICACIONES Y TRANSPORTES & 20234,4 & 0,0 & 0,0 & 20234,4 \\
\hline Servicio Postal Mexicano & 968,9 & 0,0 & 0,0 & 968,9 \\
\hline Telecomunicaciones de México & 431,0 & 0,0 & 0,0 & 431,0 \\
\hline Aeropuertos y Servicios Auxiliares & 9248,7 & 0,0 & 0,0 & 9248,7 \\
\hline Caminos y Puentes Federales de Ingresos y Servicios Conexos & 8665,9 & 0,0 & 0,0 & 8665,9 \\
\hline Otros & 919,9 & 0,0 & 0,0 & 919,9 \\
\hline EDUCACIÓN PÚBLICA & 52999,7 & 1781,7 & $(0,1)$ & 54780,7 \\
\hline Universidad Nacional Autónoma de México & 6993,9 & 665,7 & 0,0 & 7659,6 \\
\hline Consejo Nacional de Ciencia y Tecnología & $(2,1)$ & 0,0 & 0,0 & $(2,1)$ \\
\hline Colegio Nacional de Educación Profesional Técnica & 2264,6 & 28,9 & 0,0 & 2293,5 \\
\hline Comisión de Operación y Fomento de Actividades Académicas del IPN & 558,0 & 8,8 & 0,0 & 566,8 \\
\hline Instituto Nacional de Infraestructura Física Educativa & 18295,7 & 0,0 & 0,0 & 18295,7 \\
\hline
\end{tabular}




\begin{tabular}{|c|c|c|c|c|}
\hline DEPENDENCIAS Y ORGANISMOS & $\begin{array}{l}\text { SALDO } \\
2010\end{array}$ & $\begin{array}{c}\text { MOV. } \\
\text { PRESUPUESTARIO }\end{array}$ & $\begin{array}{c}\text { MOV. } \\
\text { POR AJUSTE }\end{array}$ & $\begin{array}{l}\text { SALDO } \\
2011\end{array}$ \\
\hline Patronato de Obras e Instalaciones del ION & 2485,2 & 0,0 & 0,0 & 2485,2 \\
\hline Consejo Nacional de Fomento Educativo & 14747,4 & 95,7 & $(0,1)$ & 14843,0 \\
\hline Universidad Autónoma Metropolitana & 2306,3 & 133,5 & 0,0 & 2439,8 \\
\hline Otros & 5350,7 & 848,5 & 0,0 & 6199,2 \\
\hline SALUD & 7497,3 & 1021,8 & 0,0 & 8519,1 \\
\hline IMSS - Oportunidades & 957,9 & 0,0 & 0,0 & 957,9 \\
\hline Hospitales & 1544,3 & 381,5 & 0,0 & 1925,8 \\
\hline Institutos & 4199,8 & 640,3 & 0,0 & 4840,1 \\
\hline Otros & 795,3 & 0,0 & 0,0 & 795,3 \\
\hline MEDIO AMBIENTE Y RECURSOS NATURALES & 20768,2 & 4593,2 & $(133,9)$ & 25227,5 \\
\hline Comisión Nacional Forestal & 20727,0 & 4573,8 & $(133,9)$ & 25166,9 \\
\hline Instituto Nacional de Tecnología del Agua & 41,2 & 19,4 & 0,0 & 60,6 \\
\hline DESARROLLO SOCIAL & 98,3 & 0,7 & 0,0 & 99,0 \\
\hline Instituto Nacional de las Personas Adultas Mayores & 98,3 & 0,7 & 0,0 & 99,0 \\
\hline AGRICULTURA, GANADERÍA, DESARROLLO RURAL, & 11966,0 & 143,2 & $(49,0)$ & 12060,2 \\
\hline \multicolumn{5}{|l|}{ PESCA Y ALIMENTACIÓN } \\
\hline Universidad Nacional de Chapingo & 10374,3 & 52,5 & 0,0 & 10426,8 \\
\hline Instituto Nacional de Investigaciones Forestales y Agrícolas & 704,2 & 90,7 & $(99,7)$ & 695,2 \\
\hline Colegio de Posgraduados & 531,2 & 0,0 & 29,2 & 560,4 \\
\hline Otros & 356,3 & 0,0 & 21,5 & 377,8 \\
\hline CONSEJO NACIONAL DE CIENCIA Y TECNOLOGÍA & 3334,0 & 17,5 & 0,0 & 3351,5 \\
\hline Centros e Institutos de Investigación & 3334,0 & 17,5 & 0,0 & 3351,5 \\
\hline OTRAS DEPENDENCIAS & 741,6 & 100,0 & 0,0 & 841,6 \\
\hline Varias & 741,6 & 100,0 & 0,0 & 841,6 \\
\hline
\end{tabular}

Fuente: Gobierno Federal del Estado Mexicano 2011, pág. 447.

La elaboración de los objetivos estratégicos de desarrollo, presentados en el Plan Nacional de Desarrollo y en los Planes Estatales, no se encuentran alineados, lo cual da como resultado una empresa deficiente y poco competitiva.

Las empresas públicas tienen como fuente de ingresos la recaudación de impuestos, la prestación de servicios públicos y la producción de bienes, a través del uso y explotación de recursos naturales propiedad del Estado. La ciudadanía está obligada a cuestionar la actuación de las personas a cargo de la administración pública, considerados como funcionarios y servidores públicos.

La aprobación y promulgación de las leyes en los últimos dos años ha tenido como propósito el demarcar responsabilidades en los servidores públicos, así como delimitar lo que se considera como una falta administrativa no grave. Aspectos que van desde el uso indebido de papelería, adquirida con recursos públicos, hasta el desvío de recursos financieros.
Se ha generado un marco jurídico robusto, por lo que, ahora se debe de aplicar para sancionar a los servidores públicos que infrinjan la ley, o caigan en el uso indebido de recursos materiales y financieros, propiedad de empresas públicas, provocando deficiencia en las empresas que administran.

Falta generar una cultura organizacional dentro de las empresas públicas que logre el compromiso de los servidores públicos para obtener resultados eficientes y que se refleje en el crecimiento de la productividad; falta participación de la sociedad para la rendición de cuentas de los administradores de las Empresas Públicas.

\section{REFERENCIAS BIBLIOGRÁFICAS}

Abusada, R., Cusato, A., \& Pastor, C. (2008). Eficiencia del Gasto en el Perú. Lima: Instituto Peruano de Economía. Obtenido de https://www.mef.gob.pe/contenidos/pol_econ/documentos/eficiencia_del_gasto_en_ el_peru.pdf 
Álvarez, A. (1991). Empresas Estatales y Privatización. Lima: APOYO.

CEPAL (2007) La corrupción y la impunidad en el marco del desarrollo en América Latina y el Caribe: un enfoque centrado en derechos desde la perspectiva de las Naciones Unidas. Lima: CEPAL. Obtenido de: https://www. cepal.org/es/publicaciones/6144-la-corrupcion-la-impunidad-marco-desarrollo-america-latina-caribe-un-enfoque

Diario Oficial de la Federación. (2018). Relación de Entidades Paraestatales de la Administración Pública Federal. Obtenido de: www.dof.gob.mx/nota_detalle. php codigo $=5404049 \&$ fecha $=14 / 08 / 2015$

Gobierno Federal del Estado Mexicano. (2011). Reporte de Información Financiera por el ejercicio 2011, Estados Financieros y Presupuestarios básicos. Obtenido de: http://www.apartados.hacienda.gob.mx/contabili$\mathrm{dad} /$ documentos/informe_cuenta/2011/documentos/ r04/r04d05.pdf

Fondo Nacional de Financiamiento de la Actividad Empresarial. (2017). Memoria Anual. Recuperado desde https://www.fonafe.gob.pe/centrocorporativo
Instituto Mexicano para la Competitividad. (2019). Índice de Riesgos de Corrupción: El sistema mexicano de contrataciones públicas. Obtenido de: https://imco. org.mx/articulo_es/indice-riesgos-corrupcion-sistema-mexicano-contrataciones-publicas $/$ ?gclid $=\mathrm{Cj0K}$ CQjwoInnBRDDARIsANBVyARBwCpaOaMSyi_trz6UX0dACvKzjWtjIDVoWdqSsbG-K8LU21SL4dEaAoXNEALw_wcB

Ministerio de Economía y Finanzas. (2017). Tomos Cuenta General de la República 2017. Lima. MEF. Obtenido de: https://www.mef.gob.pe/contenidos/ conta_publ/2017/empresas_publicas2017_tomo2.pdf

Ministerio de Economía y Finanzas. (2017). Programa de Mejora Continua de la Gestión de las Finanzas Públicas. Obtenido de: http://www.cooperacionsuiza.pe/ wp-content/uploads/2019/03/fs-pmc-final.pdf

Petróleos Mexicanos. (2019). Cuarto Informe Trimestral 2018. Obtenido de: http://www.pemex.com/ri/finanzas/Reporte\%20de\%20Resultados\%20no\%20Dictaminados/Reporte\%204T18.pdf

Secretaría de Hacienda y Crédito Público. (2017). Finanzas Públicas e Introducción al PbR (Material del curso). Universidad Nacional Autónoma de México, México D.F. 